\title{
Modelo de avaliaçáo de áreas urbanas para receber projetos integrados de revitalização e mobilidade sustentável
}

Raquel Felix. Universidade Federal de Itajubá, Itajubá, Brasil.

Daniela Riondet-Costa. Universidade Federal de Itajubá, Itajubá, Brasil. Josiane Palma-Lima. Universidade Federal de Itajubá, Itajubá, Brasil.

RESUmo | O objetivo deste trabalho é elaborar um modelo de priorização de áreas urbanas para projetos integrados de revitalização e mobilidade sustentável, em base aos princípios do Desenvolvimento Orientado ao Transporte (TOD). Foi desenvolvido um modelo de avaliação espacial multicritério, que posteriormente foi aplicado e validado na cidade de Itajubá-MG, Brasil. O trabalho incorporou, ainda, o uso de Funçôes Fuzzy, possibilitando a normalização e agregação de critérios qualitativos e quantitativos no Sistema de Informação Geográfica (SIG). Como resultado, mapas com índices de prioridades mostraram áreas importantes para receber os projetos de revitalização e mobilidade e urbana, indicando que existem trechos contínuos na região central da área de estudo, o que possibilita a sua implementação. $\mathrm{O}$ modelo pode auxiliar os tomadores de decisão a justificar investimentos em áreas prioritárias diante a inconveniente limitação de recursos, visando sempre melhorias na qualidade de vida da população.

PALAVRaS CHAVE | desenvolvimento sustentável, gestão urbana, mobilidade.

ABSTRACT | The objective of this work is to develop a prioritization model of urban areas for integrated revitalization and sustainable mobility projects, based on the principles of Transit Oriented Development (TOD). A spatial multicriteria evaluation model was developed, which was later applied and validated in Itajuba City, Brazil. The work also used Fuzzy Functions to normalize the criteria, following with the aggregation of qualitative and quantitative criteria in the Geographic Information System (GIS). The maps with priority indexes showed important areas to receive revitalization and mobility and urban projects, indicating that there are continuous parts in the central region of the study area, which enables their implementation. The model can help decision-makers to justify investments in priority areas in view of the inconvenient limitation of resources, always aiming to improve the quality of life of the population.

KEYWORDS | sustainable development, urban management, mobility. 


\section{Introduçáo}

$\mathrm{Na}$ segunda metade do século xx houve um processo de urbanização acelerado em cidades brasileiras devido ao êxodo rural, crescimento populacional e industrialização, sobrecarregando a infraestrutura urbana. Esses fenômenos alteraram as dinâmicas socioespaciais, provocando expansão das periferias urbanas, com a formação de habitaçôes irregulares em locais isolados e a consequente exclusão social. Atualmente, um dos principais desafios do desenvolvimento sustentável é a integração e o convívio entre os vários elementos que compóem as cidades, como as condições de acesso, as condições ambientais e as diferenças sociais. Um exemplo de uma situação avessa ao desenvolvimento sustentável são os espaços públicos destinados somente para vagas de estacionamento ou grandes viadutos, mostrando a priorização dos transportes motorizados no padrão estrutural das cidades e prejudicando a qualidade de vida da população (Antonson, Hrelja \& Henriksson, 2017).

Projetos novos ou de revitalização do ambiente urbano construído envolvem diversos interesses, que podem ser econômicos, sociais, ambientais, culturais e/ou políticos. Porém, muitas vezes os interesses são conflitantes e precisam ser trabalhados em prol do desenvolvimento sustentável (Alcántara Vasconcellos, 2010; Jacobs, 2011; Rolnik \& Klink, 2011). O desenvolvimento sustentável, por sua vez, pode ser alcançado com uma abordagem ampla de planejamento, que considera todos os elementos urbanos para promover a cidadania e condiçóes adequadas de habitação, trabalho, circulação e lazer. As recentes políticas públicas brasileiras, como o Estatuto das Cidades (2001) e a Política Nacional de Mobilidade Urbana Sustentável (2012), contemplam diretrizes em defesa do direito à cidade e a o acesso digno aos serviços públicos de qualidade, a favor de uma gestão mais democrática com a participação popular (Hidalgo \& Huizenga, 2013; Machado \& Lima, 2015). Nesse contexto, o sistemas de transportes e a mobilidade urbana participam das estratégias de gestáo, contribuindo para o crescimento inteligente das cidades (Cervero, 2013; Litman, 2017b; Mello \& Portugal, 2017; Sakamoto \& Lima, 2016; Waddell, 2011).

Com propósito de contribuir com estratégias práticas no Brasil, o Instituto de Políticas de Transporte e Desenvolvimento (ITDP, 2013) desenvolveu um manual em base aos princípios essenciais que norteiam o Desenvolvimento Orientado ao Transporte (Transit Oriented Development - TOD). Esta abordagem de desenvolvimento tem se destacado como o conceito que melhor articula as relaçóes entre o uso do solo e modos de transportes sustentáveis (Thomas \& Deakin, 2008; ITDP, 2017). Incentiva o uso de transportes sustentáveis, os modos não motorizados (ativos) e os coletivos, como alternativa ao transporte individual motorizado, bem como o uso do solo misto e cidades compactas para diminuir os deslocamentos diários (Carlton, 2009; Loo \& du Verle, 2016; Nasri \& Zhang, 2014).

Estratégias políticas, a maioria das vezes, são palpadas em restriçôes orçamentárias. Por este motivo, se faz necessário a priorização de projetos ou dos locais que irão receber melhorias. Por outro lado, devido a diversidade de usos do solo, nem todas as áreas urbanas estão aptas a receber projetos de mobilidade urbana. É um problema complexo e interdisciplinar, que envolve vários atores e fatores 
conflitantes. As metodologias de decisão que envolvem múltiplos critérios (Multiple-Criteria Decision Analysis - MCDA) são adequadas para a modelagem deste tipo de problema, permitindo a estruturação sistemática que facilita a tomada de decisão (Dragićević, Lai \& Balram, 2015; Jelokhani-Niaraki \& Malczewski, 2015; Malczewski \& Jackson, 2000; Veronesi, Schito, Grassi \& Raubal, 2017). Quando outras ferramentas são combinadas, tais como Sistemas de Informação Geográfica e lógica fuzzy, o modelo se torna ainda mais robusto (Vahdat, Smith \& Amiri, 2014; Wang, Duanmu, Lahdelma \& Li, 2018).

Portanto, o objetivo do trabalho é desenvolver um modelo de priorização de áreas urbanas para projetos integrados de revitalização e mobilidade sustentável, em base aos princípios do ToD (Desenvolvimento Orientado ao Transporte). Os conceitos do TOD foram utilizados neste trabalho para definir os indicadores do modelo de priorização de áreas urbanas. Seus objetivos contribuem com o desenvolvimento de uma cidade conectada, competitiva, eficiente, segura e com uma visão comum que aponta para o desenvolvimento urbano sustentável (Curtis, 2012).

A metodologia é composta pela associação de um método de Análise de Decisão Multicritério (MCDA) com o uso Sistema de Informação Geográfica (sIG), possibilitando a estruturação dos fatores de decisão e a análise espacial.

As políticas públicas de mobilidade sustentável e revitalização se concretizam quando se conhecem as realidades locais e as necessidades da população (Jacobs, 2011; Lima, Lima \& Silva, 2014; Rodrigues da Silva et al., 2015; Un-Habitat, 2013; Wegener, 2013). O modelo de priorização foi validado através de um estudo de caso na cidade de Itajubá no sul de Minas Gerais, Brasil, mais especificamente, em áreas limítrofes ao Rio Sapucaí, porém o modelo é facilmente adaptado e replicado para outros locais. O modelo pode auxiliar os tomadores de decisão a justificar investimentos em áreas prioritárias diante a inconveniente limitação de recursos, visando melhoria da qualidade de vida da população.

\section{Revisão da literatura}

O desenvolvimento dos sistemas de transportes contribuiu com o crescimento horizontal das cidades, em razão da facilidade de locomoção dos veículos em longas distâncias (Ratner \& Goetz, 2013). Contribuíram, também, para um padrão de atividades e uso do solo mais dispersos, afetando negativamente o acesso as atividades diárias por meio dos modos ativos. A crescente quantidade de viagens intensifica os congestionamentos, reduzindo a eficiência e funcionalidade dos modos motorizados (Hidalgo \& Huizenga, 2013; Hermida, Hermida, Cabrera \& Calle, 2015; Litman, 2017a).

Uma forma de minimizar os impactos negativos dos transportes é planejando o crescimento das cidades de uma forma mais sustentável. Cervero (2013) comenta que é crescente o interesse em estudos sobre estratégias para desenvolver cidades sustentáveis, que de acordo com Roseland (1997), é o tipo mais durável de assentamento que o ser humano é capaz de construir, com um padrão de vida aceitável sem causar profundos prejuízos ao ecossistema. Apesar deste conceito estar relacionado principalmente com a questáo ambiental, a busca pela sustentabilidade deve ser 
tratada nos seus três pilares, ambiental, econômico e social. Entretanto, a adaptação de cidades para que fiquem mais eficientes e sustentáveis é um processo de longo prazo que requer um esforço não só político, mas também da população (Rego, Nacarate, Perna \& Pinhate, 2013).

Lynch (1981) introduziu inicialmente o conceito de cidades eficientes em seu livro A Theory of Good City Form, propondo uma teoria normativa, que relaciona o valor de uma cidade às suas características espaciais. Nessa teoria, bens, serviços, lugares e informaçóes devem ser acessíveis com tempo, distância e esforço mínimos. Nessa mesma linha, com o intuito de minimizar o impacto dos deslocamentos diários na vida das pessoas, vários estudos têm sido realizados por meio dos conceitos do Desenvolvimento Orientado ao Transporte- TOD (Carlton, 2009; Curtis, 2012; Ratner \& Goetz, 2013, Nasri \& Zhang, 2014). O TOD encoraja o desenvolvimento de comunidades compactas, com uso misto do solo, com rápido acesso aos vários modos de transportes. Esses conceitos foram traduzidos pelo Instituto de Políticas de Transporte e Desenvolvimento (ITDP, 2013) como forma de incentivar o setor público e a iniciativa privada, à transformar o padrão de planejamento e o desenho urbano (Mello \& Portugal, 2017). Foram colocados na forma de manual de apoio, com indicativos de que os espaços urbanos devem ser projetados para acolher pessoas, atividades, edificaçóes e equipamentos públicos em áreas integradas ao resto da cidade por um transporte público de qualidade e facilmente conectadas a pé ou por bicicleta.

A integraçáo entre o crescimento ordenado das cidades junto ao suporte dos sistemas de transportes serve para compor planos estratégicos que garantem eficiência e qualidade na mobilidade urbana como incremento na qualidade de vida. Contribui ainda com a mudança de paradigmas no desenvolvimento urbano (Carlton, 2009; Loo \& du Verle, 2016). Os princípios que compóe o manual do TOD (ITDP, 2013) e que foram base de apoio para o desenvolvimento desse trabalho tem como base: (i) Caminhar; (ii) Pedalar; (iii) Conectar; (iv) Transporte Coletivo; (v) Misturar; (vi) Adensar; (vii) Compactar e (viii) Mudar. Quando as cidades são moldadas segundo os sete princípios antes citados, ocorre uma mudança natural de comportamento e transporte individual motorizado se torna em grande parte desnecessário à vida cotidiana. As viagens a pé, de bicicleta e pelo transporte de alta capacidade ficam mais fáceis e convenientes. Grande parte do recurso escasso e valioso que é o espaço urbano pode ser retomado das vias e dos estacionamentos que já não serão mais necessários e ser realocado a usos mais produtivos social e economicamente.

A seleção de áreas dentro do limite urbano para projetos de mobilidade é um problema de decisáo complexo pois depende de um conjunto grande de alternativas, critérios e de atores envolvidos no processo. A gestão territorial e dos sistemas de transportes separadamente apresentam problemas específicos que requerem soluçôes que nem sempre são convergentes. Entretanto, existem técnicas que podem auxiliar na estruturação desses problemas, compostos por padrôes de comportamento do meio urbano e especificidades de cada região. Métodos de análise de 
decisão multicritério (MCDA) tem sido bastante utilizados na escolha de alternativas diante de um conjunto de fatores conflitantes (Aydin \& Kahraman, 2012; Engau, Moffattm \& Dyk, 2015; Malczewski, 2006; Mardani et al., 2015), com várias aplicaçôes relacionando uso do solo e transportes (Hawas, Hassan \& Abulibdeh, 2016; Machado \& Lima, 2015; Mosadeghi, Warnken, Tomlinson \& Mirfenderesk, 2015; Taleai, Sliuzas \& Flacke, 2014). Entre os vários métodos existentes (Bana e Costa \& Vansnick., 1994; Lima, Ramos \& Fernandes Júnior, 2009; Saaty, 1990), optou-se por trabalhar com o Processo Hierárquico Analítico AHP (Analytic Hierarchy Process) proposto por Saaty (1990), pois é uma alternativa para que os diversos pontos de vista e os diversos juízos de valores possam ser unificados e tratados cientificamente (Barbosa, 2012). A eficiência do método consiste na simplicidade e habilidade para identificar a qualidade das respostas, a partir de uma análise de consistência, onde é calculado o vetor prioridade em cada matriz de comparação (Javadian, Shamskooshki \& Momeni, 2011; Saaty, 1990)

O planejamento e a decisão no ambiente urbano são por natureza um problema espacial. Para auxiliar neste processo vários autores abordam a integração de MCDA com Sistemas de Informação Geográfica (Chang, Parvathinathan \& Breeden, 2008; Feizizadeh, Jankowski \& Blaschke, 2014; Sakamoto \& Lima, 2016). Esta associação de método e ferramenta é um modo de combinar informaçóes de vários critérios para desenvolver índices de avaliação, gerando diferentes cenários e subsídios para a tomada de decisóes por gestores da administração pública e especialistas (Arefiev, Terleev \& Badenko, 2015; Malczewski, 2006; Requia, Roig, Koutrakis \& Rossi, 2016).

\section{Metodologia}

A pesquisa foi adaptada às etapas de um processo de tomada de decisão e de avaliação multicritério, esquematizadas no fluxograma apresentado na Figura 1. A etapa 1 iniciou-se com o referencial teórico, que abordou os principais temas para entendimento do problema de pesquisa. Na sequência foram selecionados os critérios que descrevem características relacionadas ao planejamento sustentável do uso do solo integrado aos transportes em base ao TOD. Uma vez definidos, os critérios foram estruturados de maneira hierárquica em grupos pertencentes a uma mesma área de abrangência. Para finalizar a etapa 1 foram desenvolvidas Matrizes de Comparação Par a Par (Método AHP) e aplicadas a um grupo de avaliadores (especialistas) para que os mesmos participassem com seus julgamentos. As planilhas foram então, encaminhadas eletronicamente a onze especialistas da área. 
FIGURA I | Fluxograma das etapas da pesquisa

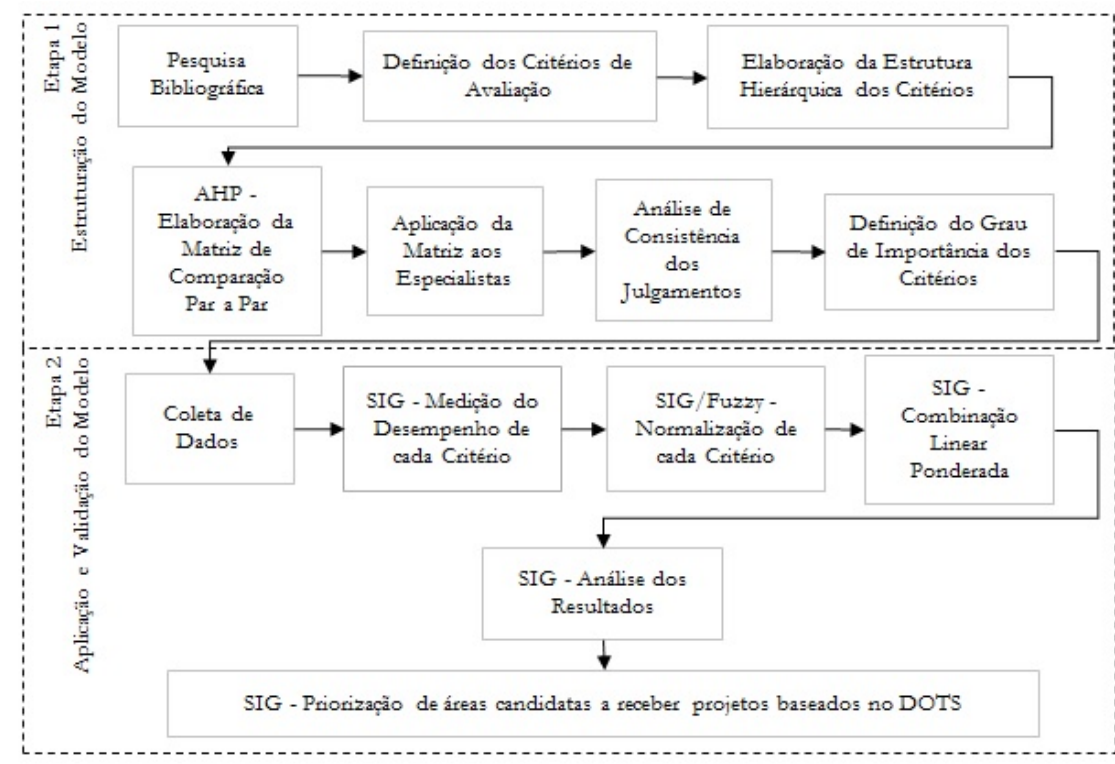

FONTE ELABORAÇão PRÓPRIA

O conjunto de avaliadores contou com pesquisadores da UNIFEI (Universidade Federal de Itajubá) e gestores do município, no qual participaram profissionais da Secretaria de Planejamento Urbano, Secretaria de Meio Ambiente, Departamento de Trânsito e Câmara de Vereadores. Os especialistas definiram o grau de importância e prioridades de cada critério no modelo de decisão, o que possibilitou o cálculo dos pesos para cada critério.

$\mathrm{Na}$ etapa 2 o modelo foi aplicado em um estudo de caso na cidade de Itajubá, Brasil. Inicialmente foi realizada a coleta de dados, para identificação das condiçóes atuais da regiáo de estudo. O banco de dados georreferenciados foi elaborado no SIG e possibilitou as análises espaciais de desempenho dos critérios por meio de mapas temáticos, seguido da combinação dos vários mapas (Combinação Linear Ponderada), considerando os pesos identificados na etapa anterior. A etapa 2 foi finalizada com a análise dos resultados da priorização das áreas para receber projetos de acordo com os critérios analisados.

\section{Modelo de priorizaçáo}

A etapa de modelagem consistiu inicialmente na identificação dos critérios de avaliação que abrangem o planejamento sustentável do uso do solo integrado aos transportes, por meio da revisão da literatura. Dos princípios do TOD (ITDP, 2017), foram definidos critérios estratégicos que visam o planejamento urbano com a construção de bairros compactos, que proporcionam a população espaços públicos seguros e ativos. 
A estrutura hierárquica foi organizada em diferentes níveis de agregação, em base ao Processo Analítico Hierárquico (AHP - Analytic Hierarchy Process). Os critérios que compóe o modelo de decisão estão descritos no Tabela 1 , bem como a descrição, a sua métrica e objetivo da priorização.

\section{TABELA I $\mid$ Descrição dos critérios}

\begin{tabular}{|c|c|c|}
\hline CRITÉRIO & DESCRIÇẪO E MÉTRICA & OBJETIVO \\
\hline $\begin{array}{l}\text { classes de Uso do } \\
\text { Solo }\end{array}$ & $\begin{array}{l}\text { Identifica o uso de áreas urbanas para as diversas atividades, tais } \\
\text { como, trabalho, comércio, lazer, educação, religioso, cultura e } \\
\text { esportes e misto. Classificaçáo de uso do solo. }\end{array}$ & $\begin{array}{l}\text { Maximiza } \\
\text { diversidade de } \\
\text { usos }\end{array}$ \\
\hline $\begin{array}{l}\text { Densidade } \\
\text { Populacional }\end{array}$ & $\begin{array}{l}\text { Considera a distribuiçấo da população em uma determinada } \\
\text { área. Populaçáo residente em uma determinada área. }\end{array}$ & $\begin{array}{l}\text { Maximiza } \\
\text { densidades }\end{array}$ \\
\hline Áreas Adensáveis & $\begin{array}{l}\text { Áreas previstas na legislação onde futuramente serão adensadas. } \\
\text { Delimitação das regiốes denominadas "Zonas de Adensamento" } \\
\text { indicadas no Plano Diretor. }\end{array}$ & $\begin{array}{l}\text { Maximiza zonas } \\
\text { de adensamento }\end{array}$ \\
\hline $\begin{array}{l}\text { Acesso ao } \\
\text { Transporte Coletivo }\end{array}$ & $\begin{array}{l}\text { Considera os transportes por ônibus urbano ou outros modos } \\
\text { coletivos. Distâncias entre as áreas de estudo e pontos de } \\
\text { ônibus. }\end{array}$ & $\begin{array}{l}\text { Maximiza a } \\
\text { Proximidade aos } \\
\text { pontos de ônibus }\end{array}$ \\
\hline $\begin{array}{l}\text { Acesso ao } \\
\text { Transporte Ativo }\end{array}$ & $\begin{array}{l}\text { Considera os transportes náo motorizados, como o modo a pé } \\
\text { e por bicicleta. Distâncias entre áreas de estudo e os locais com } \\
\text { calçadas. }\end{array}$ & $\begin{array}{l}\text { Maximiza a } \\
\text { Proximidade às } \\
\text { calçadas }\end{array}$ \\
\hline Segurança Pública & $\begin{array}{l}\text { Considera os locais inseguros, propícios à revitalização para } \\
\text { redução da criminalidade. Índice de criminalidade. }\end{array}$ & $\begin{array}{l}\text { Maximiza } \\
\text { recorrência de } \\
\text { criminalidade }\end{array}$ \\
\hline Segurança Viária & $\begin{array}{l}\text { Considera os acidentes de trânsito que envolvem pedestres e } \\
\text { ciclistas. Determinado pela recorrência de acidentes viários em } \\
\text { um local. }\end{array}$ & $\begin{array}{l}\text { Maximiza } \\
\text { recorrência de } \\
\text { acidentes }\end{array}$ \\
\hline Áreas Alagáveis & $\begin{array}{l}\text { Identifica as áreas propensas ao alagamento em épocas chuvosas. } \\
\text { Delimitação das áreas de alagamentos. }\end{array}$ & $\begin{array}{l}\text { Maximiza } \\
\text { incidência de } \\
\text { alagamentos }\end{array}$ \\
\hline Áreas Arborizadas & $\begin{array}{l}\text { Identifica locais com arborização que promovem o conforto } \\
\text { ambiental. Delimitação das áreas com presença de árvores. }\end{array}$ & $\begin{array}{l}\text { Maximiza } \\
\text { presença de } \\
\text { árvores }\end{array}$ \\
\hline Declividade & $\begin{array}{l}\text { Considera a inclinação do terreno em relação à horizontal. } \\
\text { Cálculo da inclinaçáo do terreno, em porcentagem. }\end{array}$ & $\begin{array}{l}\text { Minimiza incli- } \\
\text { naçáo }\end{array}$ \\
\hline $\begin{array}{l}\text { Afastamento do } \\
\text { Tráfego Intenso }\end{array}$ & $\begin{array}{l}\text { Proximidade do sistema viário com fluxo intenso de veículos } \\
\text { automotores. Distâncias das vias de tráfego intenso até as áreas } \\
\text { de estudo. }\end{array}$ & $\begin{array}{l}\text { Maximiza } \\
\text { distância das vias } \\
\text { principais }\end{array}$ \\
\hline $\begin{array}{l}\text { Afastamento das } \\
\text { Indústrias }\end{array}$ & $\begin{array}{l}\text { Proximidade às indústrias poluidoras. Distâncias das indústrias } \\
\text { até as áreas de estudo. }\end{array}$ & $\begin{array}{l}\text { Maximiza } \\
\text { distância de } \\
\text { indústrias }\end{array}$ \\
\hline
\end{tabular}

FONTE ELABORAÇão PRÓPRIA

A estrutura do modelo é composta por dois diferentes grupos, denominados "Questóes Ambientais" e "Questôes Sócio Territoriais".

O primeiro grupo do $1^{\circ}$ nível, Questôes Ambientais avaliam os impactos positivos e negativos que enfatizam o conforto ambiental, considerando as "Condiçóes de Relevo" e "Qualidade do Ar e Ruído". O agrupamento Condiçóes do relevo, 2 nível, que possui os critérios áreas alagáveis, áreas arborizadas e declividade, representa as principais condiçóes ambientais de relevo que influenciam no processo de urbanização. Locais em situação de risco ambiental, não devem ser considerados para novas edificaçóes, mas podem ser selecionados para revitalização e projetos 
de transportes. As áreas arborizadas são locais capazes de promover um conforto ambiental, pois a presença de árvores proporciona o equilíbrio térmico, em função do sombreamento da superfície. Os espaços arborizados também contribuem para o melhor aproveitamento da infraestrutura para integraçáo social, tais como parques e praças que propiciam o uso de modos de deslocamento sustentáveis. A declividade de um terreno identifica as regióes acidentadas e planas, características que influenciam diretamente na escolha pelo modo de transporte. Alguns autores citam que vias com grande inclinação são frequentemente evitadas por ciclistas e pedestres e, que a preferência por vias planas (de baixa declividade) é maior entre os usuários diários de transporte ativo (Beheshtitabar et al., 2014). Yang e Mesbah (2013) afirmam que náo existe consenso sobre o limite de declividade a partir do qual a via é considerada inadequada para o ciclismo ou caminhada, uma vez que a tolerância aos trechos de subida está diretamente relacionada à preparo físico e disposição do pedestre ou ciclista.

O agrupamento Qualidade do Ar e Ruído, 2o nível, tem o objetivo principal de verificar locais que possuem um grande impacto ambiental de poluição atmosférica e ruído. O sistema viário e o fluxo de veículos são importantes indicadores de poluição atmosférica, uma vez que são caracterizados como fontes móveis e considerados principais emissores dos poluentes nas áreas urbanas. Estudos comprovam que a intensidade do tráfego e o ano da frota dos veículos estão relacionados diretamente a qualidade e a dispersâo dos poluentes, assim também outros fatores como tempo de exposição, relevo e ventos (Habermann, Paula, Medeiros \& Gouveia, 2011; Hoek, Brunekreef, Goldbohm, Fischer \& van den Brandt, 2002). A literatura possui vários estudos que discutem a relação direta entre a exposição aos ambientes poluídos e seus efeitos adversos a saúde, principalmente o ruído e poluição atmosférica causadores de doenças que afetam o sistema nervoso, respiratório e cardiovascular. Pesquisas caracterizam os casos de exposição aos impactos ambientais negativos gerados pelo ruído e poluentes atmosféricos, as áreas distantes até $100 \mathrm{~m}$ das infraestruturas viárias por onde trafegam as fontes móveis (Arbex et al., 2012; Requia et al., 2016).

O segundo Grupo do $1^{\circ}$ nível, Questóes Sócio Territoriais, avalia as relaçóes da sociedade no território considerando fatores de "Uso do Solo", "Transporte Sustentável" e "Segurança”.

No agrupamento Uso do solo, $2^{\circ}$ nível, foram consideradas questóes de uso e ocupação do solo, como densidade populacional, classes de uso do solo e áreas adensáveis, de acordo com o Plano diretor do município. O critério Classes de uso do solo identifica as diferentes atividades desempenhadas pela população no espaço urbano, que muitas vezes estão espaçadas umas das outras. Para minimizar esse problema, um dos princípios do TOD defende a redução das distâncias entre as atividades intensificando o uso misto do solo em uma mesma região. O critério Densidade populacional analisa a distribuição espacial da população. Altas densidades indicam maior demanda pelo sistema de transporte, justificando, na maioria das vezes, a alocação de recursos em projetos de transporte público coletivo (ITDp, 2017). McFarlane (2016) comenta que lugares da cidade com mais pessoas caminhando ou andando de bicicleta podem proporcionar interação social e encorajar outras pessoas a usar os 
modos de transportes sustentáveis, pois elas se sentem mais seguras e confiantes de andar a pé e de bicicleta. É importante comentar que políticas de segurança pública devem ser prioridades também nestes locais.

No agrupamento Transporte Sustentável, 20 nível, a importância do transporte coletivo é evidenciada nos princípios do TOD, principalmente quando se refere às questóes de acessibilidade. $\mathrm{O}$ ToD trata do acesso ao transporte coletivo como uma das maneiras de o tornar uma opção mais atrativa e segura. As melhorias na qualidade do modo coletivo, junto à revitalização do espaço urbano e à integração com outros deslocamentos sustentáveis contribui diretamente na redução de viagens individuais motorizadas. A importância do transporte ativo é evidenciada nos princípios do TOD, principalmente por orientar o planejamento de comunidades urbanas sustentáveis focada nos pedestres e ciclistas. Os modos de transportes sustentáveis minimizam as emissóes de poluentes locais e de efeito estufa, promovem bem-estar social ao contribuir para a saúde pública, prevenindo doenças respiratórias e reduzindo os índices de obesidade.

Ainda dentro de questôes sócio territoriais, o agrupamento Segurança aborda as condiçóes de segurança pública e segurança viária. Esta questáo contribui para a qualidade de vida da populaçáo e interfere diretamente nas escolhas pelo modo de transportes. Locais inseguros que passaram por processo de revitalização, se tornaram mais seguros, atraindo as pessoas e incentivando caminhadas e ciclistas (Segadilha \& Sanches, 2014). Foi inserido neste grupo por ser um importante fator social. Estudos indicam a existência da correlação entre as taxas de violência nos espaços urbanos e as desigualdades sociais, econômicas e carência de infraestrutura de serviços de necessidade básica (Ramão \& Wadi, 2010). Segundo Tilahun, Levinson e Krizek (2007), a segurança oferecida nas vias urbanas é um dos fatores mais relevantes quando o usuário faz a escolha da rota para o deslocamento, mesmo quando comparado ao tempo de viagem, condiçôes climáticas e relevo irregular.

Para avaliar a importância relativa entre os critérios adotou-se o método de comparação aos pares, em base ao AHP, a partir da escala de nove níveis de Saaty. As avaliaçóes ocorreram por meio da matriz de comparação elaboradas em planilhas no Excel, encaminhadas eletronicamente a onze especialistas da área. O conjunto de avaliadores contou com pesquisadores da UNIFEI e gestores do município, no qual participaram profissionais da Secretaria de Planejamento Urbano, Secretaria de Meio Ambiente, Departamento de Trânsito e Câmara de Vereadores.

Na Figura 2 são apresentados o modelo de priorização com os critérios e os seus respectivos pesos. Constatou-se a diversidade de julgamentos entre os critérios decorrido das diferentes especialidades dos avaliadores. Contudo o método desenvolvido por Saaty (1990) possibilitou analisar a consistência do julgamento dos avaliadores. 


\section{FIgura 2 Modelo de Priorização}

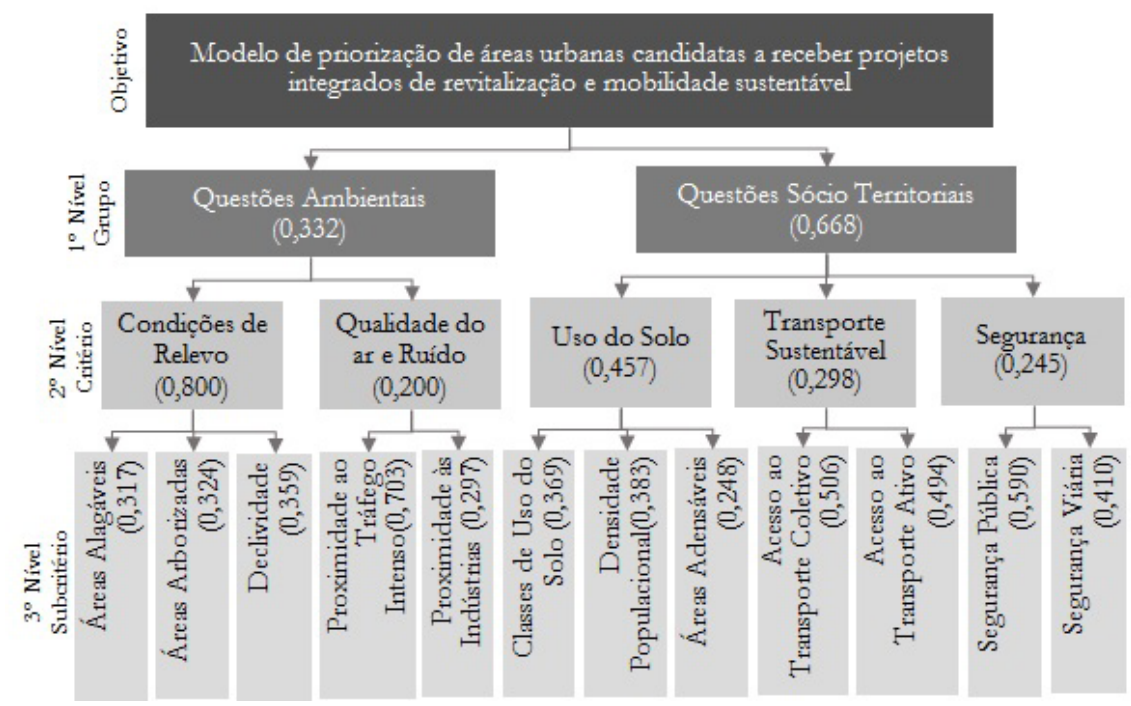

FONTE ELABORAÇão PRÓPRIA

\section{Aplicação e análise dos resultados}

Entende-se por consistência, a coerência lógica na atribuição dos valores na comparação, principalmente quando são comparados três ou mais critérios na matriz. Por fim, foi possível reunir todas as avaliaçóes individuais dos especialistas, verificando o grau de importância entre os critérios e o cálculo do peso médio de cada critério.

O modelo foi aplicado no município de Itajubá, situado no sul do Estado de Minas Gerais (MG) no Brasil. O município encontra-se na Serra da Mantiqueira, onde atinge a altitude de 1.746 metros no seu ponto mais alto e de 830 metros no ponto mais baixo. Sua área urbana encontra-se na altitude média de 842 metros, com 294,83 km² de extensão, sendo $86 \%\left(254,03 \mathrm{~km}^{2}\right)$ de área rural e $14 \%$ $\left(40,80 \mathrm{~km}^{2}\right)$ de área urbanizada. A população em 2010 foi calculada em 90.658 habitantes, numa taxa anual de crescimento de 1,26\% habitantes por ano (Instituto Brasileiro de Geografia e Estatística - IBGE, 2010). Assim como em diversos municípios, o histórico da ocupação em Itajubá iniciou-se às margens planificadas dos rios, principalmente em razáo da topografia local que, segundo IBGE (2010) ela é caracterizada como ondulada montanhosa: $10 \%$ plana, $12 \%$ ondulada e $78 \%$ montanhoso.

O objeto de estudo foi definido pela área delimitada por um buffer, de raio de 50 metros, a partir das margens do Rio Sapucaí, dentro do limite urbano. A escolha da área de estudo se deu baseada na importância histórica de ocupação que se consolidou no entorno deste rio. Vale ressaltar que se trata de uma regiáo que interliga diversos bairros do município e mesmo náo dispondo de infraestruturas adequadas é constantemente utilizada para deslocamentos a pé ou por bicicletas, ou como lazer 
e recreação. O Código Florestal Brasileiro (Lei Federal no 12.651, de 25 de maio de 2012) delimita como Área de Preservação Permanente as faixas marginais do Rio Sapucaí em 50 metros a partir da borda da calha do leito regular, admitindo que a largura média entre as bordas se enquadra na faixa de 10 a 50 metros.

A aplicação do modelo teve início com a coleta dos dados para cada critério pertencente ao $3^{\circ}$ nível da estrutura hierárquica (conforme a Figura 2), determinando as condiçóes atuais dos critérios na área de estudo. $\mathrm{O}$ banco de dados e as análises espaciais foram gerados no software de geoprocessamento ArcGIS 10.4.1. A partir da análise espacial foi necessário a padronização dos dados, uma vez que cada critério foi mensurado considerando suas próprias características para o dimensionamento. Então, foram aplicadas funçóes de normalização diretamente do sIG, que possibilitam converter valores numéricos e linguísticos em uma escala única comparável. Neste trabalho foram adotadas funçôes fuzzy crescente ou descrente, para normalizaçáo dos valores na escala numérica de 0 a 1 , determinadas de acordo com a natureza do critério.

Os critérios foram agregados em ambiente SIG, em base a estrutura definida no modelo de priorização e nos níveis hierárquicos (Figura 2). A expressão que agrega os critérios normalizados corresponde a uma Combinação Linear Ponderada (WLC - Weighted Linear Combination), que permite atribuir os pesos para cada critério e avalia-los na combinação dos critérios de um mesmo nível.

Inicialmente, foi realizada a combinação dos critérios ( $2^{\circ}$ nível) agregando seus sub-critérios levando em consideração os respectivos pesos (Figura 3, 4 e 5) e para finalizar, foi feito o agrupamento combinando os critérios no $1^{\circ}$ nível (Figura 6), gerando o Mapa do Índice Global de Prioridades.

As Figuras 3 e 4 apresentam os critérios relacionados com as Questôes Ambientais, mostrando, para fins de exemplo, o processo inicial de construção dos dados primários, a normalização de cada um dos critérios desagregados e por fim o mapa combinado de Condiçóes do Relevo e Qualidade do Ar e Ruído, respectivamente. O mapa das "Condiçôes de Relevo" (2ºnível, Figura 3) considera as interferências físicas que influenciam no processo de urbanização. Portanto, este critério combina os subcritérios "Áreas Alagáveis", "Áreas Arborizadas" e "Declividade”. Observa-se que mesmo com a atribuição de pesos semelhantes aos critérios, as "Áreas Arborizadas" atuaram com maior influência no índice de prioridades. Ou seja, as áreas com maior prioridade nos bairros periféricos são arborizadas, apresentam pouca variação de declividade e estâo inseridas em área de inundação.

O mapa de "Qualidade do ar e Ruído" (2ºnível, Figura 4) considera as distâncias das principais fontes geradoras de poluição sonora e atmosférica, sendo o resultado da combinação entre os subcritérios "Afastamento do Tráfego Intenso" e "Afastamento das Indústrias". Foram priorizadas as áreas afastadas do tráfego pesado e das indústrias, visto que esses locais não são agradáveis ao uso de modos ativos sustentáveis de transporte. Nota-se que grande parte da área de estudo apresenta valores baixos de prioridade, em consequência da proximidade da principal rodovia de acesso à cidade, que permeia o rio Sapucaí em quase toda a sua extensão e junto a ela também estão localizadas as indústrias de maior impacto. 
FIGURA 3 | Combinação para “Condiçôes de Relevo”

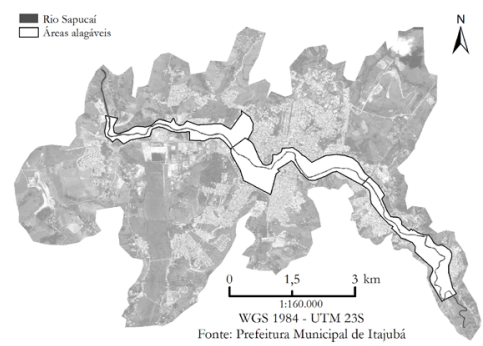

a.1) Áreas alagáveis

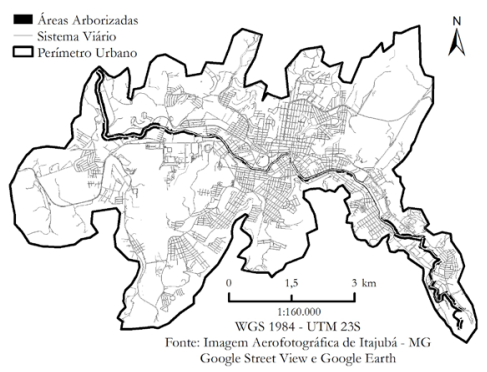

b.1) Áreas arborizadas

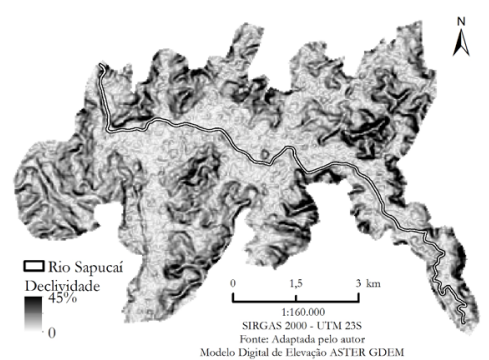

c.1) Declividade

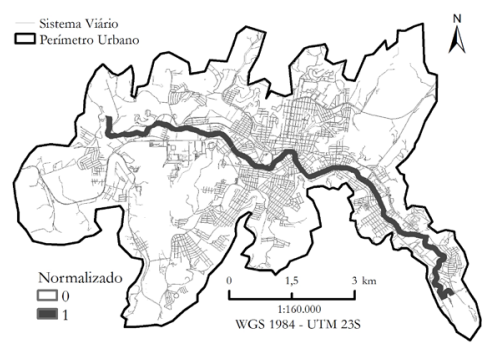

a.2) Áreas alagáveis normalizado

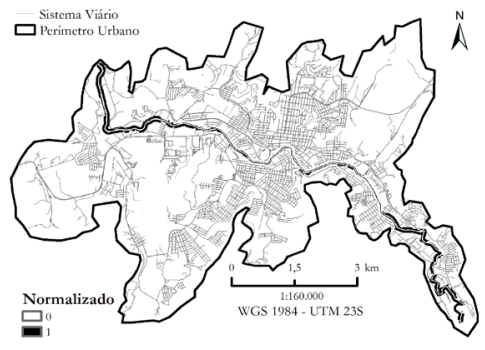

b.2) Áreas arborizadas normalizado

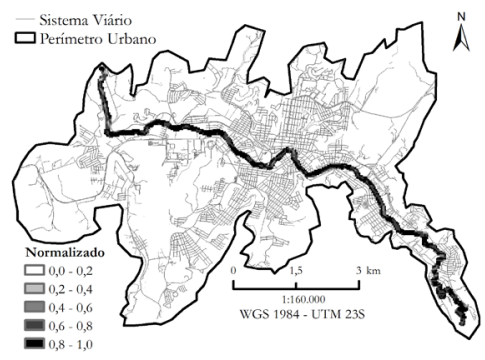

c.2) Declividade normalizado

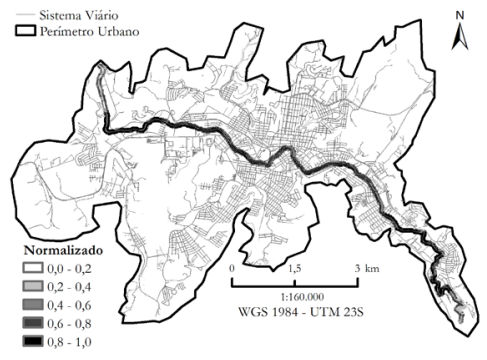

d) Condições do Relevo

FONTE ELABORAÇão PRÓPRIA 
FIGURA 4 | Combinação para “Qualidade do Ar e Ruído”
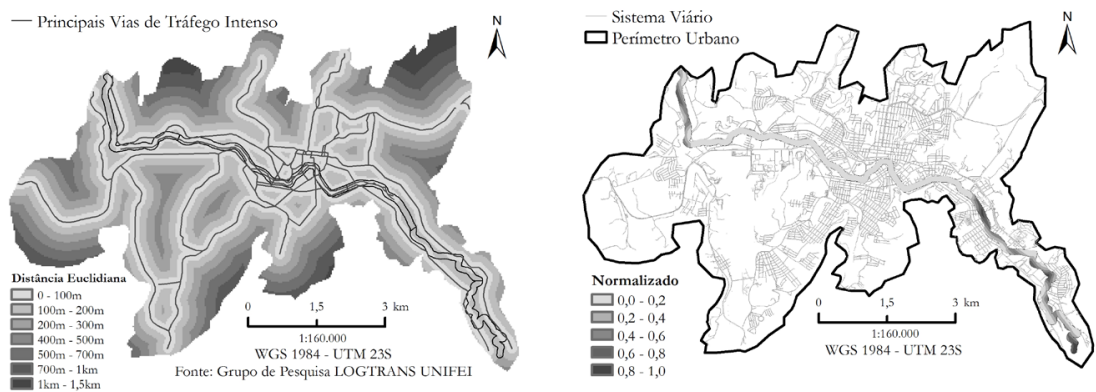

a.1) Afastamento do tráfego intenso

a.2) Afastamento do tráfego intenso normaliza
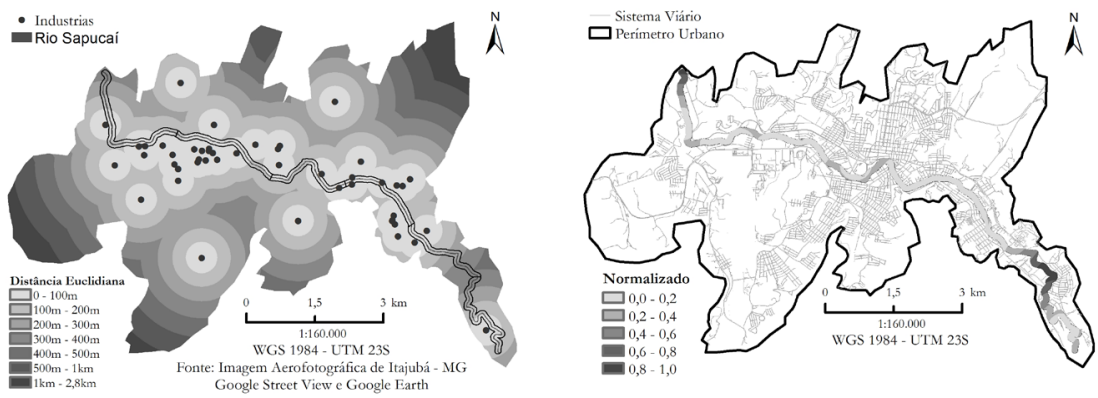

b.1) Afastamento de industrias

b.2) Afastamento de industrias normalizado

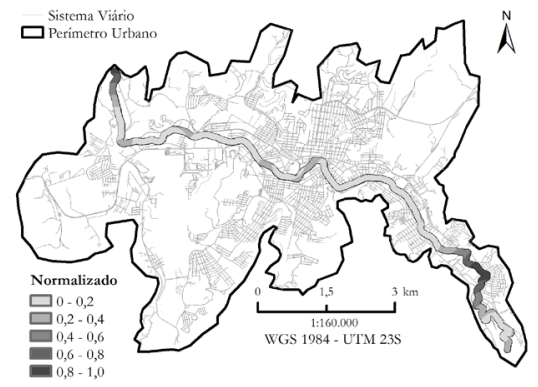

c) Qualidade do ar e ruído

FONTE ELABORAÇão PRÓPRIA

A Figura 5 apresenta os mapas combinados dos critérios relacionados com as Questôes Sócio Territoriais. Inicialmente, o critério "Uso do Solo" (2º nível, Figura 5a) teve a finalidade de combinar e avaliar os subcritérios "Classes de uso do solo", "Densidade populacional” e "Áreas Adensáveis, ou seja, os tipos de atividades realizadas na região estudada, bem como a distribuição da população em relação às atividades exercidas no meio urbano. Na Figura 5a observa-se a escala ponderada 
através de cores que indicam as regióes prioritárias. Ainda de acordo com o uso do solo, as áreas prioritárias se estendem em toda regiáo central condizente com as áreas adensadas e com maior diversidade de usos.

\section{FIgURA 5 | Combinação para "Questóes Sócio Territoriais"}

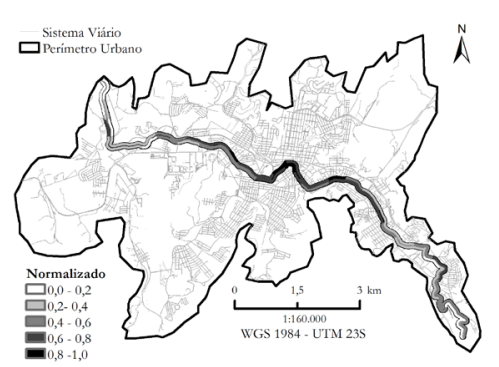

a) "Uso do solo"

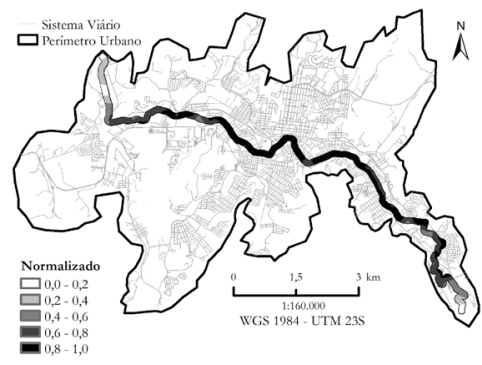

b) "Transporte Sustentável"

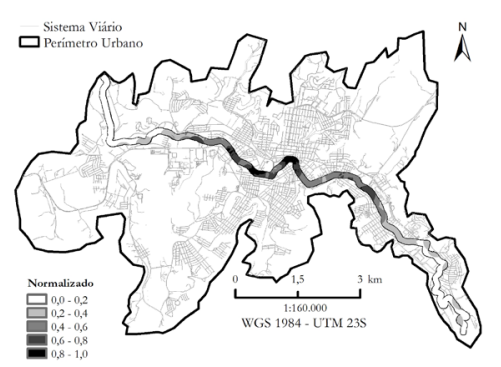

c) "Segurança"

FONTE ELABORAÇão PRÓPRIA

$\mathrm{Na}$ Figura 5b é apresentado o resultado da combinação dos subcritérios "Acesso ao Transporte Coletivo" e "Acesso ao Transporte Ativo". O "Transporte Sustentável”, que integra estes dois subcritérios, trata das condiçôes do sistema viário que afetam diretamente a escolha do usuário por utilizar os modos de transportes sustentáveis. Observa-se que grande parte da área de estudo obteve um índice de prioridade alto, o que representa ser uma área com proximidade às calçadas e aos pontos de ônibus, propícias à integração dos transportes sustentáveis.

Na Figura 5c é apresentado o mapa referente a "Segurança", resultado da combinação dos subcritérios "Segurança Pública" e "Segurança Viária, que considera o fato da escolha por modos sustentáveis estar diretamente ligada a sensação de segurança nas vias urbanas, uma vez que áreas degradas e inseguras não são atrativas ao transporte ativo. Observa-se que as áreas prioritárias localizadas na regiáo central merecem maior atenção do poder público, com projetos de revitalização e mobilidade urbana que tragam maior iteração social, o que consequentemente auxilia nos indicadores de segurança pública e viária. Os locais que apresentam os maiores valores normalizados devem ser priorizados, pois sáo possíveis áreas que requerem melhorias na infraestrutura para recuperação da segurança. 
Depois de realizar a combinação dos vários subcritérios para gerar os mapas dos critérios do $2^{\circ}$ nível, foi realizada a combinação dos critérios, gerando os mapas dos grupos no $1^{\circ}$ nível, apresentado na Figura 6.

O grupo denominado "Questóes Sócio Territoriais" (1º nível) combina as características da infraestrutura urbana que favorecem a integração social para aumentar a qualidade de vida da população, resultado da combinação dos critérios "Uso do Solo", "Transporte Sustentável" e "Segurança”, conforme é apresentado na Figura 6a. Observa-se que as áreas priorizadas no mapa recebem grande influência do critério "Uso do Solo" quando comparado aos outros critérios, devido ao fato de a área central (mais prioritária) da cidade possuir muitos locais com uso misto, fazendo elevar o índice quando multiplicado por um valor alto de grau de importância e ainda corroborando com ambientes bastante adensados.

O segundo grupo do $1^{\circ}$ nível, denominado "Questóes Ambientais" resulta da combinação entre os critérios "Condiçóes de Relevo" e "Qualidade do ar e Ruído", conforme apresentado na Figura 7. As "Questóes Ambientais” relacionam critérios que caracterizam o conforto ambiental que podem propiciar mudanças no comportamento social, como a escolha por modos ativos ou coletivos, por exemplo. A partir da combinação ponderada, constata-se a compensaçáo dos critérios com a priorização das áreas centrais no município.

A compensação é verificada, uma vez que as áreas periféricas ao centro ao mesmo tempo que apresentam maior prioridade considerando as "Condiçóes de Relevo", também apresentam distâncias muito próximas as fontes geradoras de poluição sonora e atmosférica o que acarreta a diminuição da prioridade.

\section{FIGURA 6 | Combinação dos Grupos (10 Nível)}

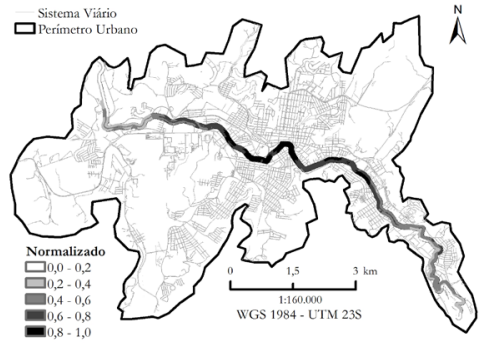

a) "Questões Sócio Territoriais"

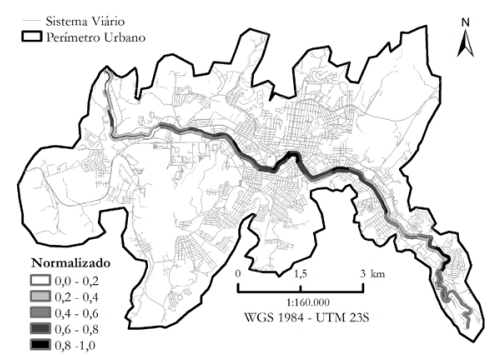

b) "Questões Ambientais"

FONTE ELABORAÇão PRÓPRIA

Por fim, depois de realizada a combinação dos critérios por nível hierárquico, gerando os mapas dos grupos de critérios no $1^{\circ}$ e $2^{\circ}$ nível, foi realizada a última combinação de critérios, gerando o mapa final (global) de prioridades. Portanto, foram combinados os mapas dos grupos "Questôes Sócio Territoriais" e "Questôes Ambientais" para obter o índice global de priorização das áreas no entorno do Rio Sapucaí, apresentado na Figura 7. 
FIgura 7 | Índice Geral de Priorização de Áreas

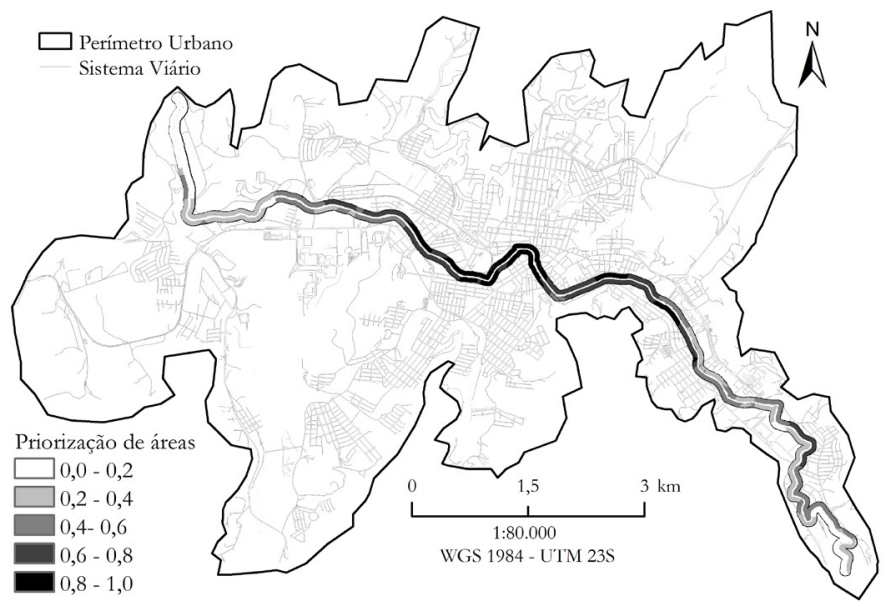

FONTE ELABORAÇão PRÓPRIA

Nota-se que o maior trecho priorizado apresenta integralidade e conecta os bairros centrais às zonas industriais do município. Também são identificados outros trechos menores, que possibilitam a análise dos tomadores de decisão na priorizaçáo da aplicação de projetos diante de recursos limitados, visando à integração futura entre os diferentes trechos. Esta é uma conclusão importante, pois de acordo com estudos anteriores e os conceitos do TOD (ITDP, 2017), projetos de mobilidade urbana sustentáveis devem permitir o uso dos vários modos de forma conjunta, com trechos contínuos que proporcionem a integridade, necessária a segurança e o bem-estar da população.

As questôes relevantes consideradas para a priorização dessas áreas no município, contempladas nos critérios abordados tem impacto direto no uso da terra e nas decisóes dos usuários para acessar o sistema de transportes. De acordo com os trechos demarcados como prioridade (Figura 7), como exemplo nota-se a coerência em implantar projetos que visem os transportes sustentáveis nas regióes mais adensadas, como mencionado por McFarlane (2016). Esses projetos podem ser tanto de iluminação, quanto de construçáo e ampliação de calçadas, ciclovias, parques lineares ou melhorias na qualidade do transporte coletivo, ou seja, projetos que promovam o bem estar e qualidade de vida da população.

\section{Conclusốes e discussóes}

O objetivo deste trabalho foi propor um modelo de priorização de áreas urbanas para projetos integrados de revitalização e mobilidade sustentável, em base aos princípios do TOD (Desenvolvimento Orientado ao Transporte). As cidades estáo propensas a sofrer com o processo de urbanizaçáo crescente, em vários setores que passam por segurança pública, saneamento, habitação e transportes. Os conceitos 
do TOD foram utilizados neste trabalho para definir os indicadores do modelo. Seus objetivos contribuem com o desenvolvimento de uma cidade conectada, competitiva, eficiente, segura. $\mathrm{O}$ uso dos conceitos abordados no ToD para a elaboração do modelo de decisão se apresentou como uma ferramenta conceitual importante para traçar as diretrizes e elaboração dos critérios de decisão, e com uma visão comum que aponta para o desenvolvimento urbano sustentável.

A modelagem se mostrou satisfatória para o objetivo proposto. Foram considerados os princípios de compactação dos centros com a promoção de usos mistos do solo e adensamento populacional, criação de espaços púbicos que intensifiquem a interação social, com priorização dos modos de transportes sustentáveis, como transporte coletivos e modos ativos, que concorda com a teoria de cidades eficientes de Lynch (1981). A estruturação do modelo foi bastante complexa, concordando com Taleai et al. (2014) e McFarlane (2016) sobre a complexidade do problema de gestáo urbana, uma vez que envolve um conjunto de fatores multidisciplinares. A associação do método de avaliação multicritério com sIG foi uma boa escolha metodológica pois permitiu a avaliação estruturada dos fatores de decisão, também evidenciado anteriormente por Malczewski (2006).

Por sua vez, o Processo Hierárquico Analítico (AHP) adotado para estruturar hierarquicamente os critérios, permitiu a avaliaçáo do grau de importância utilizando a comparação par a par, julgada por especialistas de vários setores da administração pública do município. O método AHP se mostrou uma importante ferramenta durante esse processo, pois facilitou a compreensáo do problema por parte dos especialistas e, como resultado, o modelo absorveu um conjunto de julgamentos distintos. Foi uma abordagem enriquecedora na composição do grau de importância, mas em outras situaçóes ou problemas pode ser considerada uma fragilidade de um modelo desenvolvido. As opinióes diversas foram importantes na composição do grau de importância de cada critério, pois são abrangentes no contexto de problemas complexos e da multidisciplinariedade dos critérios. O resultado dos julgamentos apontou maior importância às questóes sócio territoriais (67\%), mostrando a influência das questóes de uso do solo e transporte no processo decisório. As questôes ambientais, que abordam condiçóes de relevo e qualidade do ar e ruído ficaram com 33\% do grau de importância no processo de seleção de áreas para projetos de revitalizaçáo e mobilidade urbana. Esse julgamento reflete a abordagem de Yang \& Mesbah (2013). Áreas que muitas vezes não são propícias à habitação devido condiçôes ambientais, podem ser apropriadas para projetos alternativos de mobilidade e lazer, como por exemplo, parques urbanos com prioridade para as calçadas e ciclovias, promovendo a acessibilidade, equidade e a integração social.

$\mathrm{O}$ trabalho apresenta resultados com implicações práticas e políticas. O modelo foi aplicado na cidade de Itajubá-MG, no Brasil, no entorno do Rio Sapucaí, devido à importância dessa regiấo no processo de urbanizaçấo. A etapa de coleta de dados foi a mais demorada, tanto pela quantidade de dados adquiridos quanto pela diversidade de instituiçóes visitadas. De acordo com Sakamoto \& Lima (2016) a disponibilidade de dados é um ponto crucial em pesquisas dessa natureza. Verificou-se que as análises seriam mais precisas se estivessem disponíveis dados como, pesquisa Origem/Destino, contagem e classificação das vias, emissão de poluentes. 
Entretanto, os dados coletados possibilitaram uma análise satisfatória dos critérios e aplicação do modelo de priorização. Como resultado, o mapa de índices de prioridades apontou áreas críticas diante do processo de urbanização sem o planejamento adequado e que deveriam receber projetos de revitalização. Verificou-se também trechos lineares na região central da área de estudo, o que facilitaria a implementação de projetos de mobilidade com maior integralidade, mostrando à coerência do resultado, com os princípios do TOD. Se trata de uma região articuladora do sistema viário, que interliga vários bairros no município e carece de investimentos para revitalização e mobilidade urbana.

Cabe comentar, que o modelo não abordou os aspectos políticos e econômicos das decisóes, uma vez que o objetivo maior do modelo é verificar tecnicamente as áreas urbanas aptas a receber projetos híbridos de revitalização e mobilidade. Se trata de um modelo de auxílio a decisão e cabe aos administradores verificar diante de recursos escassos se está condizente com o cronograma da organização pública. Da mesma forma, pedidos pontuais da população também não foram considerados, uma vez que se trata de um processo mais dinâmico das necessidades e da realidade de cada região. Os vários cenários gerados, para cada grupo e ainda critérios desagregados, é um importante subsídio de auxílio à tomada de decisão aos órgãos públicos da cidade estudada. Por meio do sIg os especialistas e gestores públicos tiveram maior compreensão visual da interferência e relevância dos critérios, característica do SIG que também foi verificado anteriormente por outros autores (Feizizadeh et al., 2014; Requia et al., 2016). Os resultados podem justificar investimentos diante de recursos limitados, propondo políticas públicas mais sustentáveis, especialmente no que tange ao uso do solo e a integração com os projetos de transporte, tornando a cidade mais eficiente, garantindo ainda o direito a um espaço urbano mais justo.

\section{Agradecimentos}

Os autores agradecem ao CNPq (Conselho Nacional de Desenvolvimento Científico e Tecnológico), à fapemig (Fundação de Amparo à Pesquisa do Estado de Minas Gerais) e à CAPEs (Coordenação de Aperfeiçoamento de Pessoal de Nível Superior) pelo apoio financeiro concedido em projetos que subsidiaram o desenvolvimento deste trabalho.

\section{Referências bibliográficas}

Alcántara Vasconcellos, E. (2010). Análisis de la movilidad urbana. Espacio, medio ambiente y equidad. Bogotá, Colombia. https://doi.org/doi.org/If7432010202974

Antonson, H., Hrelja, R., \& Henriksson, P. (2017). People and parking requirements: Residential attitudes and day-to-day consequences of a land use policy shift towards sustainable mobility. Land Use Policy, 62, 213-222. https://doi.org/10.1016/j. landusepol.2016.12.022 
Arbex, M. A., Santos, U. de P., Martins, L. C., Saldiva, P. H. N., Pereira, L. A. A., \& Braga, A. L. F. (2012). A poluição do ar e o sistema respiratório. Jornal Brasileiro de Pneumologia, 38(5), 643-655. https://doi.org/10.1590/S1806-37132012000500015

Arefiev, N., Terleev, V., \& Badenko, V. (2015). Grs-based fuzzy method for urban planning. Procedia Engineering, 117(1), 39-44. https://doi.org/10.1016/j.proeng.2015.08.121

Aydin, S., Kahraman, C., \& Kaya, I. (2012). A new fuzzy multicriteria decision making approach: An application for European Quality Award assessment. Knowledge-Based Systems, 32, 37-46. https://doi.org/10.1016/j.knosys.2011.08.022

Bana e Costa, C. A., \& Vansnick, J. C. 1994. масветн: An interactive path towards the construction of cardinal value functions. International Transactions in Operational Research, 1(4), 489-500. https://doi.org/10.1016/0969-6016(94)90010-8

Barbosa, L. F. S. 2012. Formulação de um Modelo Multicritério para a Avaliação de Acessibilidade na concepção de prédios. Dissertaçâo (Mestrado) Universidade Federal Fluminense. Niterói.

Beheshtitabar, E., Ríos Aguilar, S., König-Hollerwöger, D., Svatý, Z., \& Rydergren, C. (2014). Route choice modelling for bicycle trips. International Journal for Traffic and Transport Engineering, 4(2), 194-209. https://doi.org/10.7708/ijtte.2014.4(2).06

Carlton, I. (2009). Histories of transit-oriented development: perspectives on the development of the TOD concept. Working Paper 2009-02, Institute of Urban and Regional Development, University of California, Berkeley. http://iurd.berkeley.edu/wp/2009-02.pdf

Cervero, R. B. (2013). Linking urban transport and land use in developing countries. Journal of Transport and Land Use, 6(1), 7. https://doi.org/10.5198/jtlu.v6i1.425

Chang, N.-B., Parvathinathan, G., \& Breeden, J. B. (2008). Combining GIs with fuzzy multicriteria decision-making for landfill siting in a fast-growing urban region. Journal of Environmental Management, 87(1), 139-153. https://doi.org/10.1016/j. jenvman.2007.01.011

Curtis, C. (2012). Delivering the "D" in transit-oriented development: examining the town planning challenge. Journal of Transport and Land Use, 5(3), 83-99. https://doi. org/10.5198/jtlu.v5i3.292

Dragićević, S., Lai, T., \& Balram, S. (2015). GIs-based multicriteria evaluation with multiscale analysis to characterize urban landslide susceptibility in data-scarce environments. Habitat International, 45(2): 114-125. https://doi.org/10.1016/j. habitatint.2014.06.031

Engau, A., Moffattm C., \& Dyk, W. (2015) Multicriteria modeling and tradeoff analysis for oil load dispatch and hauling operations at Noble energy. Optimization and Engineering, 16(1), 73-101. https://doi.org/10.1007/s11081-014-9259-5

Feizizadeh, B., Jankowski, P., \& Blaschke, T. (2014). A GIs based spatially-explicit sensitivity and uncertainty analysis approach for multi-criteria decision analysis. Computers \& Geosciences, 64, 81-95. https://doi.org/10.1016/j.cageo.2013.11.009

Habermann, M., Paula, A., Medeiros, P., \& Gouveia, N. (2011). Tráfego veicular como método de avaliação da exposição à poluição atmosférica nas grandes metrópoles. Revista Brasileira de Epidemiologia, 14(1), 120-130. https://bit.ly/2kqZufi

Hawas, Y.E., Hassan, M. N., \& Abulibdeh, A. (2016) A multi-criteria approach of assessing public transport accessibility at a strategic level. Journal of Transport Geography, 57, 19-34. https://doi.org/10.1016/j.jtrangeo.2016.09.011 
Hermida, M. A., Hermida, C., Cabrera, N., \& Calle, C. (2015). La densidad urbana como variable de análisis de la ciudad . EURE-Revista Latinoamericana de Estudios Urbano Regionales, 41(124), 25-44. https://doi.org/10.4067/S0250-71612015000400002

Hidalgo, D., \& Huizenga, C. (2013). Implementation of sustainable urban transport in Latin America. Research in Transportation Economics, 40(1), 66-77. https://doi. org/10.1016/j.retrec.2012.06.034

Hoek, G., Brunekreef, B., Goldbohm, S., Fischer, P., \& van den Brandt, P. A. (2002). Association between mortlaity and indicators of traffic-related air pollution in the Netherlands: a cohort study. The Lancet, 360, 1203-1209.

IBGE - Instituto Brasileiro de Geografia e Estatística. (2010). Censo Demográfico. Características da população e dos domicílios. http://www.ibge.gov.br/home/

ITDP - Institute for Transportation and Development Policy. (2017). The TOD Standard. https:// www.itdp.org/tod-standard/

ITDP - Instituto de Políticas de Transporte e Desenvolvimento. (2013). Padrão de Qualidade TOD. http://itdpbrasil.org.br/padrao-de-qualidade-tod/

Jacobs, J. (2011). Morte e vidas de grandes cidades - The death and life of great american cities $\left(3^{\circ}\right)$. Tradução por Carlos S. Mendes Rosa, Revisão técnica de Cheila Aparecida Gomes Bailão. São Paulo: wmF Martins Fontes.

Javadian, M., Shamskooshki, H., \& Momeni, M. (2011). Application of sustainable urban development in environmental suitability analysis of educational land use by using AHP and Gis in Tehran. Procedia Engineering, 21, 72-80. https://doi.org/10.1016/j. proeng.2011.11.1989

Jelokhani-Niaraki, M., \& Malczewski, J. (2015). Decision complexity and consensus in Webbased spatial decision making: A case study of site selection problem using GIS and multicriteria analysis. Cities, 45, 60-70. https://doi.org/10.1016/j.cities.2015.03.007

Lima, J. P., Ramos, R. A. R., \& Fernandes Júnior, J. L (2009). A multicriteria approach to the prioritization of paved roads. Transportes, 17(1), 2738. http://dx.doi.org/10.14295/ transportes.v17i1.73

Lima, J. P., Lima, R. da S., \& Silva, A. N. R. da. (2014). Evaluation and selection of alternatives for the promotion of sustainable urban mobility. Procedia - Social and Behavioral Sciences, 162(Panam), 408-418. https://doi.org/10.1016/j.sbspro.2014.12.222

Litman, T. (2017a). Evaluating accessibility for transportation planning. Measuring people's ability to reach desired goods and activities. Victoria Transport Policy Institute. http://www. vtpi.org/access.pdf (January 2015).

Litman, T. (2017b). Land use impacts on transport. Victoria Transport Policy Institute, 1-85. https://doi.org/10.1007/978-3-642-54876-5

Loo, B. P. Y., \& du Verle, F. (2016). Transit-oriented development in future cities: towards a two-level sustainable mobility strategy. International Journal of Urban Sciences, 5934(November), 1-14. https://doi.org/10.1080/12265934.2016.1235488

Lynch, K. (1981). A Theory of good city form. Cambridge, MA: MIT Press.

Machado, M. H., \& Lima, J. P. (2015). Avaliação multicritério da acessibilidade de pessoas com mobilidade reduzida: um estudo na região central de Itajubá (MG). URBE. Revista Brasileira de Gestão Urbana, 7(3), 368-382. https://doi.org/10.1590/21753369.007.003.AO08 
Malczewski, J., \& Jackson, M. (2000). Multicriteria spatial allocation of educational resources: an overview. Socio-Economic Planning Sciences, 34(3), 219-235. http://dx.doi. org/10.1016/S0038-0121(99)00025-7.

Malczewski, J. (2006). GIs-based multicriteria decision analysis: a survey of the literature. International Journal of Geographical Information Science, 20(7), 703-726. https://doi. org/10.1080/13658810600661508

Mardani, A., Jusoh, A., MD Nor, K., Khalifah, Z., Zakwan, N., \& Valipour, A. (2015). Multiple criteria decision-making techniques and their applications - A review of the literature from 2000 to 2014. Economic Research-Ekonomska Istraživanja, 28(1), 516-571. https://doi.org/10.1080/1331677X.2015.1075139

McFarlane, C. (2016). The geographies of urban density: Topology, politics and the city. Progress in Human Geography, 40(5), 629-648. https://doi.org/10.1177/0309132515608694

Mello, A., \& Portugal, L. (2017). Um procedimento baseado na acessibilidade para a concepção de planos estratégicos de mobilidade urbana: O caso do Brasil. EURE Revista Latinoamericana de Estudios Urbano Regionales, 43(128), 99-126. https://doi. org/10.4067/S0250-71612017000100005

Mosadeghi, R., Warnken, J., Tomlinson, R., \& Mirfenderesk, H. (2015). Comparison of Fuzzy-AHP and AHP in a spatial multi-criteria decision making model for urban landuse planning. Computers, Environment and Urban Systems, 49, 54-65. https://doi. org/10.1016/j.compenvurbsys.2014.10.001

Nasri, A., \& Zhang, L. (2014). The analysis of transit-oriented development (TOD) in Washington, D.C. and Baltimore metropolitan areas. Transport Policy, 32, 172-179. https://doi.org/10.1016/j.tranpol.2013.12.009

Ramão, F. P., \& Wadi, Y. M. (2010). Espaço urbano e criminalidade violenta: análise da distribuição espacial dos homicídios no município de Cascavel/PR. Revista de Sociologia e Politica, 18(35), 207-230. https://doi.org/10.1590/S0104-44782010000100013

Ratner, K. A., \& Goetz, A. R. (2013). The reshaping of land use and urban form in Denver through transit-oriented development. Cities, 30, 31-46. https://doi.org/10.1016/j. cities.2012.08.007

Rego, J. A. A., Nacarate, J. P. M., Perna, L. N., \& Pinhate, T. B. (2013). Cidades Sustentáveis: Lidando com a urbanização de forma ambiental, social e economicamente sustentável. Simulação das Naçôes Unidas para Secundaristas (pp. 545-573). http://www.sinus.org. br/2013/wp-content/uploads/2013/03/17.-PNUMA-Artigo.pdf

Requia, W. J., Roig, H. L., Koutrakis, P., \& Rossi, M. S. (2016). Mapping alternatives for public policy decision making related to human exposures from air pollution sources in the Federal District, Brazil. Land Use Policy, 59, 375-385. https://doi.org/10.1016/j. landusepol.2016.09.017

Rodrigues da Silva, A. N., Azevedo Filho, M. A. N. de, Macêdo, M. H., Sorratini, J. A., da Silva, A. F., Lima, J. P., \& Pinheiro, A. M. G. S. (2015). A comparative evaluation of mobility conditions in selected cities of the five Brazilian regions. Transport Policy, 37, 147-156. https://doi.org/10.1016/j.tranpol.2014.10.017

Rolnik, R., \& Klink, J. (2011). Crescimento econômico e desenvolvimento urbano: por que nossas cidades continuam tão precárias? Novos Estudos - CEBRAP, 89(89), 89-109. https://doi.org/10.1590/S0101-33002011000100006 
Roseland, M. 1997. Dimensions of the eco-city, Cities, 14(4), 197-202. https://doi. org/10.1016/S0264-2751(97)00003-6

Saaty, T. L. (1990). How to make a decision: The analytic hierarchy process. European Journal of Operational Research, 48(1), 9-26. https://doi.org/10.1016/0377-2217(90)90057-I

Saaty, T. L. (2008). Decision making with the analytic hierarchy process. International Journal of Services Sciences, 1(1), 83. https://doi.org/10.1504/IJSSCI.2008.017590

Sakamoto, E., \& Lima, J. P. (2016). Acessibilidade em ambiente rural: uma abordagem multicritério com uso de sIg. Transportes, 24(1), 63. https://doi.org/10.14295/ transportes.v24i1.958

Segadilha, A. B. P., \& Sanches, S. da P. (2014). Identification of factors that influence cyclistś route choice. Procedia - Social and Behavioral Sciences, 160(Cit), 372-380. https://doi. org/10.1016/j.sbspro.2014.12.149

Taleai, M., Sliuzas, R., \& Flacke, J. (2014). An integrated framework to evaluate the equity of urban public facilities using spatial multi-criteria analysis. Cities, 40, 56-69, Part A. https://doi.org/10.1016/j.cities.2014.04.006

Thomas, A., \& Deakin, E. (2008). Land Use challenges to implementing transit-oriented development in China: Case study of Jinan, Shandong Province. Transportation Research Record: Journal of the Transportation Research Board, 2077, 80-86. https://doi. org/10.3141/2077-11

Tilahun, N. Y., Levinson, D. M., \& Krizek, K. J. (2007). Trails, lanes, or traffic: Valuing bicycle facilities with an adaptive stated preference survey. Transportation Research Part A: Policy and Practice, 41(4), 287-301. https://doi.org/10.1016/j.tra.2006.09.007

Un-Habitat. (2013). Planning and Design for Sustainable Urban Mobility: Global Report on Human Settlements 2013. https://bit.ly/2GOSnpv

Vahdat, K., Smith, N. J., \& Amiri, G. G. (2014). Fuzzy multicriteria for developing a risk management system in seismically prone areas. Socio-Economic Planning Sciences, 48(4), 235-248. https://doi.org/10.1016/j.seps.2014.05.002

Veronesi, F., Schito, J., Grassi, S., \& Raubal, M. (2017). Automatic selection of weights for GIs-based multicriteria decision analysis: site selection of transmission towers as a case study. Applied Geography, 83, 78-85. https://doi.org/10.1016/j.apgeog.2017.04.001

Waddell, P. (2011). Integrated land use and transportation planning and modelling: Addressing Challenges in research and practice. Transport Reviews, 31(2), 209-229. https://doi.or g/10.1080/01441647.2010.525671

Wegener, M. (2013). The future of mobility in cities: Challenges for urban modelling. Transport Policy, 29, 275-282. https://doi.org/10.1016/j.tranpol.2012.07.004

Wang, H., Duanmu, L., Lahdelma, R., \& Li, X. (2018). A fuzzy-grey multicriteria decision making model for district heating system. Applied Thermal Engineering, 128, 10511061. https://doi.org/10.1016/j.applthermaleng.2017.08.048

Yang, C., \& Mesbah, M. (2013). Route choice behaviour of cyclists by stated preference and revealed preference. Australasian Transport Research Forum 2013 Proceedings (October). http://atrf.info/papers/2013/2013_yang_mesbah.pdf 